\title{
The reliability of lung ultrasound in assessment of idiopathic pulmonary fibrosis
}

This article was published in the following Dove Press journal:

Clinical Interventions in Aging

\section{Diana Manolescu' \\ Lavinia Davidescu² \\ Daniel Traila ${ }^{3}$ \\ Cristian Oancea ${ }^{3}$ \\ Voicu Tudorache ${ }^{3}$}

'Radiology Department, University of Medicine and Pharmacy "Victor

Babes", Timișoara, Romania;

2Department of Pulmonology,

University of Medicine and

Pharmacy Oradea, Oradea, Romania;

${ }^{3}$ Department of Pulmonology,

University of Medicine and Pharmacy

"Victor Babes", Timișoara, Romania

Correspondence: Lavinia Davidescu Department of Pulmonology, University of Medicine and Pharmacy Oradea,

I Decembrie sq, nr 10, Oradea, Bihor, Romania

Tel +40 74| I4| 734

Email lavinia.davidescu@yahoo.com

\begin{abstract}
Idiopathic pulmonary fibrosis (IPF) is the severest form of idiopathic interstitial pneumonia, with a median survival time estimated at 2-5 years from the time of diagnosis. It occurs mainly in elderly adults, suggesting a strong link between the fibrosis process and aging. Although chest high-resolution computed tomography (HRCT) is currently the method of choice in IPF assessment, diagnostic imaging with typical usual interstitial pneumonia (UIP) provides definitive results in only $55 \%$, requiring an invasive surgical procedure such as lung biopsy or cryobiopsy for the final diagnostic analysis. Lung ultrasound (LUS) as a noninvasive, non-radiating examination is very sensitive to detect subtle changes in the subpleural space. The evidence of diffuse, multiple B-lines defined as vertical, hyperechoic artifacts is the hallmark of interstitial syndrome. A thick, irregular, fragmented pleura line is associated with subpleural fibrotic scars. The total numbers of B-lines are correlated with the extension of pulmonary fibrosis on HRCT, being an LUS marker of severity. The average distance between two adjacent B-lines is an indicator of a particular pattern on HRCT. It is used to appreciate a pure reticular fibrotic pattern as in IPF compared with a predominant ground glass pattern seen in fibrotic nonspecific interstitial pattern. The distribution of the LUS artifacts has a diagnostic value. An upper predominance of multiple B-lines associated with the thickening of pleura line is an LUS feature of an inconsistent UIP pattern, excluding the IPF diagnosis. LUS is a repeatable, totally radiation-free procedure, well tolerated by patients, very sensitive in detecting early changes of fibrotic lung, and therefore a useful imaging technique in monitoring disease progression in the natural course or after initiation of treatment.
\end{abstract}

Keywords: chest ultrasound, chest high-resolution computed tomography, B-lines artifacts, interstitial syndrome, interstitial lung diseases

\section{Introduction}

Idiopathic pulmonary fibrosis (IPF) is defined as a specific form of chronic, progressive fibrosing interstitial pneumonia of unknown cause. ${ }^{1}$ IPF is the most common and severe form of idiopathic interstitial pneumonia, ${ }^{1-3}$ is irreversible, with an unpredictable and variable clinical course, ${ }^{4}$ and is associated with an extremely poor prognosis. ${ }^{5-7}$

IPF occurs mainly in elderly adults, over 60 years old, with a prevalence of $>300 / 100,000$, suggesting a strong link between the fibrosis process and aging. Besides telomere shortening, mitochondrial dysfunction, loss of proteostasis, other genetic and epigenetic factors are hallmarks of the accelerated aging process. ${ }^{8}$

IPF diagnosis requires the exclusion of known causes of interstitial lung disease (ILD), environmental factors attributable to the manifested pulmonary fibrosis, or the absence of collagen vascular disease. ${ }^{9,10}$ A final diagnosis may not be achieved, even after lengthy multidisciplinary discussions, ${ }^{9}$ remaining "unclassifiable." 
Accounting for $15 \%-30 \%$ of interstitial lung diseases (ILD), ${ }^{11-13}$ IPF is an important clinical problem, with a median survival estimated at 2-5 years from the time of diagnosis. ${ }^{14,15}$ Most of the deaths are due to respiratory failure or complicating comorbidity. ${ }^{16,17}$

Although ILDs are a heterogeneous group of diseases, they all share the same inflammatory pathway and fibrous changes of interstitial space with the reconstruction of the lung structure. ${ }^{13}$

Chest high-resolution computed tomography (HRCT) is currently considered the main standard diagnostic in all ILD assessments, not only for baseline evaluation, but also for monitoring the disease and prediction of treatment efficacy. ${ }^{18}$ We have to be aware of the radioprotection issue, since the cumulative dose is $7 \mathrm{mSv}$ per examination, which corresponds with 2 years of natural light exposure. ${ }^{19}$

The diagnostic algorithm of IPF requires the presence of a typical usual interstitial pneumonia (UIP) pattern on $\mathrm{HRCT}^{20,21}$ with the presence of honeycombing and reticular abnormalities in a predominantly basal and subpleural distribution. ${ }^{22}$ The agreement between thoracic radiologists for the diagnosis of UIP/IPF based on the current guidelines is moderate. ${ }^{23}$

The presence of bilateral lower lobe subpleural reticular abnormalities without honeycombing and without atypical features is recognized as a possible UIP pattern. ${ }^{18}$ In patients with this pattern, surgical lung biopsy (or cryobiopsy) with evidence of UIP is the method of choice to confirm the diagnosis of IPF. ${ }^{22}$ In most of the cases, this approach is

A


"Ring-down" artifacts

B

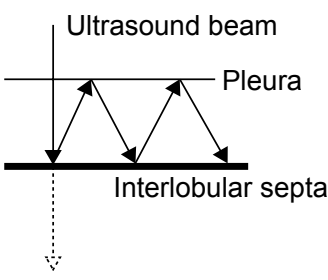

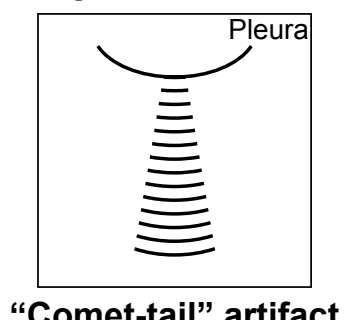

“Comet-tail” artifact difficult because of the clinical condition, comorbidities, or patient refusal.

\section{Lung ultrasound (LUS) evaluation in IPF}

The main advantage of LUS examination is that it is a noninvasive diagnostic procedure, without any exposure to irradiation. It is repeatable, cost-effective, and well tolerated by patients. ${ }^{24}$

Ultrasound (US) abilities are based not only on the capacity to visualize real images of disorders ${ }^{25,26}$ (eg, direct visualization of pleural effusion or lung consolidations) but also on analyzing various artifacts. ${ }^{27}$

Based on the basic physical principle of sound waves, the remarkable potential of US in the study of lung parenchyma lies in its ability to detect alterations in parenchymal density resulting from the loss of alveolar air with or without an increase of interstitial fluids. ${ }^{28}$

Scanning a normally aerated lung permits visualization of the entire chest wall from the skin to pleura. Below the pleura, due to the thin interlobular septa and no alteration of alveolar space, the air creates an interface with the pleura and the soft tissues of the chest wall because of differences in acoustic impedance (air is an acoustic barrier) (Figure 1). ${ }^{29}$

The image corresponding to the normal lung is obtained by artifacts that represent the chest wall's reflections below the pleural line ("mirror effect" of the lung or A-lines). ${ }^{27}$

A-lines represent horizontal lines visualized at constant intervals in the zone of artifacts and represent multiple reflections of the pleural line. Some authors ${ }^{30}$ use the term

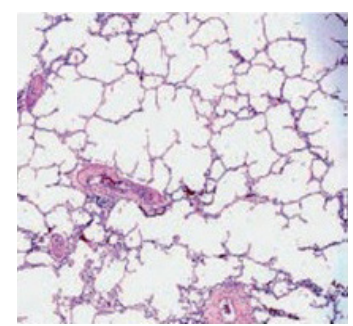
Normal lung

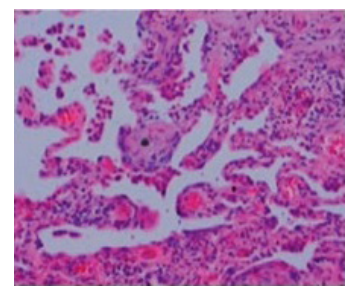

Fibrotic lung

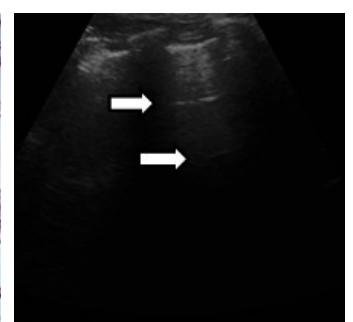

A-lines

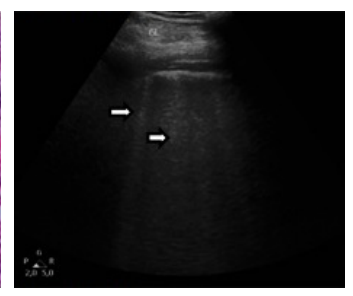

B-lines

Figure I The physical and anatomic basis of echo lung comets.

Notes: (A) Normally aerated lung with A-lines artifact as reflection of pleura line. (B) Reflections of the ultrasound beam by the thickened interlobular septa proved comettail artifact (B-lines) in patients with IPF. Modified from Am J Cardiol, 93(I0), Jambrik Z, Monti S, Coppola V, et al, Usefulness of ultrasound lung comets as a nonradiologic sign of extravascular lung water, 1265-1270, Copyrgiht (2004), with permission from Elsevier. ${ }^{29}$

Abbreviation: IPF, idiopathic pulmonary fibrosis. 


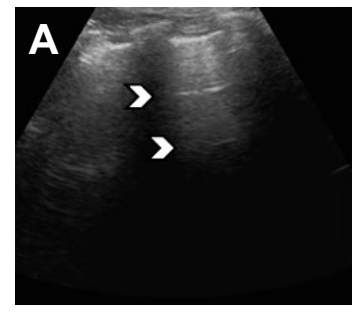

Normal lung

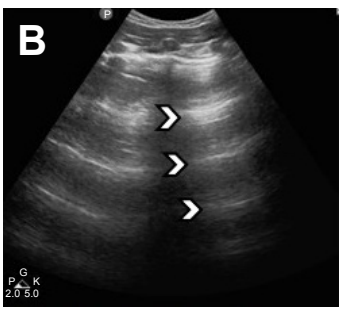

Emphysematous lung

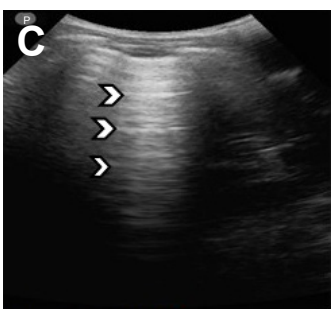

Honeycombing

Figure 2 Aspect of A-lines (arrows) in (A) normal lung, (B) emphysematous lung, and (C) IPF with honeycombing.

Abbreviation: IPF, idiopathic pulmonary fibrosis.

"multiple A-lines," due to the increase in the amount of subpleural air. These occur in the case of emphysematous changes or honeycombing and may be a prognostic factor of severity in patients with IPF (Figure 2).

The LUS assessment of IPF is carried out by the detection and quantification of B-lines. ${ }^{31}$

B-lines are long, well-defined, laser-like, hyperechoic artifacts arising from the pleural line. ${ }^{32,33}$ They are generated by the reflection of the US beam from thickened subpleural interlobular septa detectable in between the lung intercostal spaces (LIS). ${ }^{34,35}$ Patients with heart failure have diffuse multiple B-lines due to thickening of interlobular septa by water, as a first sign of pulmonary congestion. ${ }^{36}$

An increased amount of extravascular water in the alveolar compartment has a direct correlation with the number of B-lines, with $>10$ lines per LIS. ${ }^{32}$ Multiple B-line artifacts merging into one broad vertical artifact, generating the "white lung," are an LUS finding in predominant alveolar patterns ${ }^{37}$ corresponding to ground glass opacities (GGOs) on HRCT (Figure 3).

A horizontal hyperechoic line, half a centimeter below the rib line, is defined as the pleura line (Figure 4). This separates the chest wall from the lung. In healthy adults or in patients with pulmonary congestion, this line is thin and not $>2 \mathrm{~mm}$. In the case of a fibrotic lung, this line is thickened $>3 \mathrm{~mm}$, with an irregular fragmented appearance.

Interstitial syndrome is defined by more than three comettail artifacts arising from the pleura line, visible in a frozen image in a longitudinal scan, with a distance no more than $7 \mathrm{~mm}$ between two lines, in both lungs. ${ }^{38}$

In IPF, interstitial syndrome is generated by the accumulation of collagen in the subpleural interlobular septa. LUS is

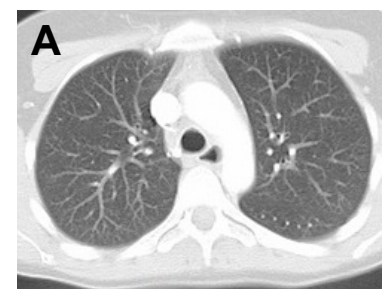

Normal transparency

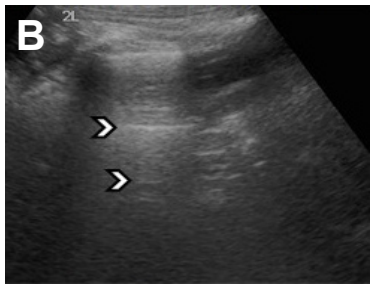

Normal aerated lung

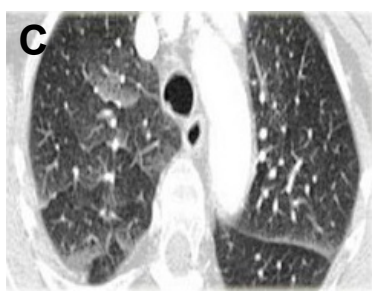

Extensive GGO

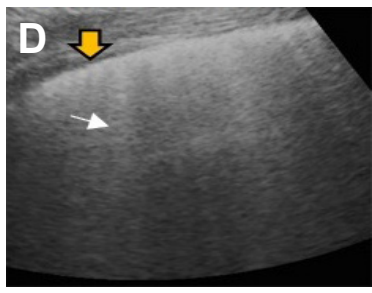

White lung

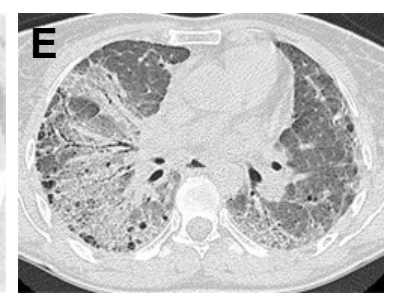

Fibrotic NSIP

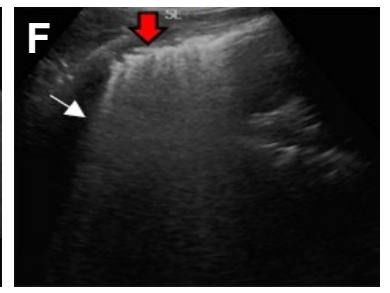

Fibrotic white lung



Fibrotic UIP

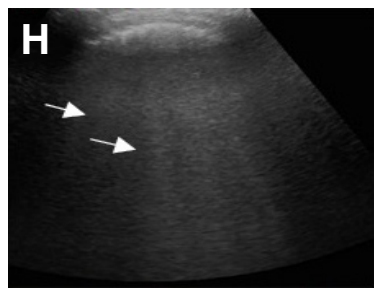

Fibrotic interstitial syndrome

Figure 3 HRCT-LUS correlation in normal lung, pulmonary congestion, rheumatoid lung disease, idiopathic pulmonary fibrosis.

Notes: (A) Normal lung transparency; (B) A-lines (arrow head) reverberation artifacts from normal interlobular septa; (C) heart failure with extensive ground glass opacities; (D) "white lung" due to alveolar syndrome with multiple long, vertical hyperechoic B-line artifacts (thin arrow) arising from a smooth pleura line (yellow arrow); (E) NSIP fibrotic lung with extensive ground glass opacities and traction bronchiectasis due to pulmonary volume loss; $(\mathbf{F})>10$ B-lines in one IC with thickened, fragmented pleura line (red arrow) in a fibrotic alveolar syndrome (thin arrow shows B-line artifact); (G) UIP pattern with subpleural, basal reticular abnormalities, traction bronchiectasis, and honeycombing; (H) B-lines with irregular thick pleura line (thin arrows show B-line artifacts).

Abbreviations: HRCT, high-resolution computed tomography; LUS, lung ultrasound; NSIP, nonspecific interstitial pattern; IC, intercostal space; UIP, usual interstitial pneumonia; GGO, ground glass opacity. 

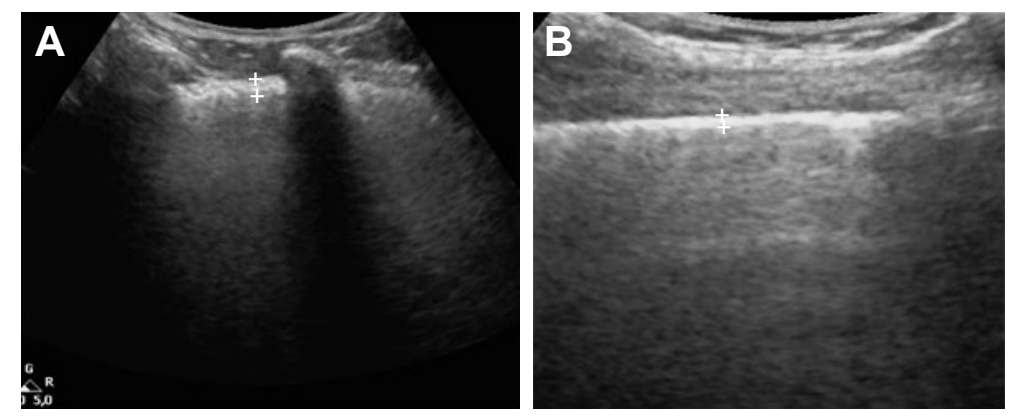

Figure 4 Pleura line appearance.

Notes: (A) Thickened (>3 mm) irregular pleura line due to subpleural fibrotic abnormalities; (B) normal thickness $(2 \mathrm{~mm})$.

an accurate tool that can differentiate between a fibrotic and cardiogenic interstitial syndrome by an assessment of pleura line thickness. ${ }^{39}$

\section{Correlations between LUS and HRCT in the evaluation of pulmonary fibrosis}

It is well known that thoracic US examination can be used to evaluate patients with pulmonary fibrotic damage in a systemic disease. From our knowledge, published studies have focused on the assessment of pulmonary fibrosis of secondary cause. Analyzed groups of patients have been compared with healthy volunteers. ${ }^{40}$ Patients with IPF were included in these studies as a part of the heterogeneous group. ${ }^{35,41}$

These studies compared the elements of pulmonary morphology (pleural line) or reverberation artifacts (B-lines) highlighted in the pulmonary US examination with the HRCT exam as the "gold standard" of the imaging diagnosis. ${ }^{40}$

In 2009, Gargani et $\mathrm{al}^{42}$ published the first comparative data between US examination and HRCT assessment of pulmonary fibrosis arguing that the identification of B-lines in the chest US examination may be an indicator of pulmonary diffuse interstitial impairment in patients with scleroderma. HRCT is the only method that evaluates the whole lung parenchyma, and not just the subpleural region. An important linear correlation between the total number of B-lines in US scores and Warrick's semiquantitative tomographic score ${ }^{43}$ was identified, with $P<0.001$ and a good match in assessing the degree of severity. B-lines provided more evidence in the diffuse form of the disease than in the limited form. The limitation of this first study was that it included a group of patients with predominantly mild and moderate forms.

In the same year, Sperandeo et $a l,{ }^{35}$ in a group of 84 patients with pulmonary fibrosis $(63 \%$ with IPF confirmed by biopsy), linked the morphologic US elements (reverberation artifacts, pleura line thickness, and the presence of subpleural cysts) with the severity degree of pulmonary fibrosis on HRCT and functional assessment. The US examination included the evaluation of all intercostal spaces (ISs) in the anterior, posterior, and axillary chest wall. An irregular pleural line with a thickness of $>3 \mathrm{~mm}$ was disposed in all the patients with fibrosis when compared to a similar group of healthy volunteers $(<2 \mathrm{~mm})$, pleural thickening being the only sonographic element detected in patients with mild forms. Moderate forms were associated with the presence of subpleural cysts, while severe forms demonstrated a reduced "lung sliding sign" (assessed in the M-mode), due to small movement of visceral pleura by subpleural fibrosis. B-lines were present in greater numbers in the moderate and severe forms.

In 2015, Sperandeo et $\mathrm{al},{ }^{44}$ in an extended study of 175 patients with systemic scleroderma, reconfirmed the correlation between pleural thickness and the extension of the tomographic grade of fibrosis, suggesting the LUS screening role. The thickness of the pleural line was assessed with a $3 \mathrm{~mm}$ cutoff. Diffuse forms are associated with a pleura thickness varying between 3 and $5 \mathrm{~mm}$, while the severe forms had a thickness $\geq 5 \mathrm{~mm}$. In 26 of the patients who had a normal HRCT examination, $3 \mathrm{~mm}$ thick pleura was identified, giving LUS increased sensitivity in the detection of incipient forms.

Hasan and Makhlouf, based on the definition of interstitial syndrome, considered a positive US examination to have $\geq 3$ B-lines per IS (LIS) in more than two bilateral lung regions or $>10 \mathrm{~B}-$ lines totally. ${ }^{41}$ This study included patients with multiple-cause pulmonary fibrosis (50\% with IPF) and assessed the correlation between the HRCT pulmonary fibrosis score evaluated by Warrick's model and the distance between two B-lines. A distance up to $3 \mathrm{~mm}$ corresponds ultrasonically with multiple B-lines, creating the "white lung" appearance encountered in ground glass modeling at the HRCT exam. In patients with predominant reticulation and honeycombing, the distance between B-lines was $7 \mathrm{~mm}$, 
resulting in the appearance of a "long rocket." Pulmonary US, supported by HRCT correlation, is a distinct tool for differentiation between alveolar and interstitial syndrome by simply measuring the distance between two B-lines.

The role of US examination in the diagnosis of incipient forms of pulmonary fibrosis is also demonstrated by Barskova et $\mathrm{al}^{39}$ in a group of patients with scleroderma. Eighty-eight percent of these patients had a positive HRCT diagnosis, $41 \%$ of them had incipient forms. The US was evaluated for the presence of B-lines and the total number was quantified. Concordance between HRCT evaluation and US was $83 \%$. In the remaining cases considered falsely positive, the US examination revealed the presence of B-lines as markers of interstitial syndrome. By excluding the cardiogenic cause of lung damage, LUS specificity in the diagnosis of incipient pulmonary fibrosis was $100 \%$. Thus, LUS is a reliable tool in evaluating the very early signs of fibrosis and screening for pulmonary involvement in ILD.

Recently, Buda et al, ${ }^{30}$ evaluating 64 patients with ILD (30\% with IPF) by HRCT and LUS, described a semiquantitative method of assessing the severity of pulmonary fibrosis. For clinical reasons, the author adjusted Warrick's tomography scale for quantification of fibrosis and proposed an alveolar index up to 4 points for the active form of disease and a fibrotic index up to 35 points for fibrotic changes. This fibrotic index is compared with the ultrasound fibrotic index. The term multiple A-lines and a blurred pleura line being introduced for the severe form of fibrosis associated with extensive reticulation and honeycombing injury on HRCT.

Even though there was a high degree of variability, these studies were convergent in terms of linear correlation between the two diagnostic imaging methods, confirming the utility of pulmonary US in assessing pulmonary fibrosis. To date, there are no data published in the literature about LUS assessment in IPF patients as a single group.

The variability of these studies consisted of the following aspects.

1. The heterogeneity of the populations of patients - these were mixed, including varied forms of ILD, especially focusing on connective tissue diseases. ${ }^{45}$ Some of the studies had a group of healthy volunteers as a comparative group. The number of patients varied from nine patients with sarcoidosis ${ }^{41}$ to 175 patients with systemic sclerosis. $^{44}$

2. Another variable element was the US examination technique. Different types of equipment were used, from parasternal (PS)-ultrasound device (USD) pocket-sized ${ }^{46}$ to performance US machines. The sensitivity of LUS considering HRCT as the method of choice in ILD assessment is around $89 \%$, without any significant variability depending on the devices used.

3. The probes used were different. In the initial studies, ${ }^{35,39,42}$ a 2.5-3.5 MHz cardiac sector transducer was used; then, a transducer with a variable frequency of 2-7 MHz was utilized ${ }^{45,47}$ Some authors also associated a linear probe with a high frequency of $8-11 \mathrm{MHz}^{30,44,48}$ for better characterization of pleural line appearance or thickness.

As possible factors might influence B-lines visualization, some caution should be exercised. ${ }^{49}$

\section{US examination protocol}

The patient is examined in the dorsal decubitus position with arms on the head for the anterior and lateral thoracic regions, and in the sitting position for the assessment of the posterior regions (Figure 5)..$^{50}$

For the anterior and lateral region of the chest wall LUS was performed assessing the second to the fifth ISs both sides on the PS, midclavicular (MC), anterior axillary (AA), and medial axillary (MA) lines, summing to a total number of 28 LIS. The seventh and eighth IS on the posterior axillary (PA) and subscapular (SS) lines, and also the second to the eighth intercostal space (IC) on the paravertebral line were assessed for the posterior chest wall region, summing to a total of 22 LIS..$^{39,42,47}$ The median time was 23 minutes for all 50 LIS. $^{47}$

Gutierrez et al found a good correlation between a reduced LUS protocol of 14 LIS (Figure 6) and the comprehensive 50 LIS protocol. ${ }^{45}$ The reduced protocol included the assessment of the second LIS along the PS line and the fourth LIS along the MC, AA, MA lines for the anterior chest wall region. For the posterior region, the eight LIS along PA, SC, and paravertebral (PV) lines were assessed. Smaller median time was observed from 23.3 to 8.6 minutes, making this 14 LIS protocol suitable for the screening of pulmonary fibrosis.

\section{LUS is reliable in differentiating between a UIP pattern and a fibrotic nonspecific interstitial pattern (NSIP)}

Based on known guidelines, an atypical HRCT finding such as extensive ground glass abnormalities is a feature of inconsistent UIP pattern and therefore excludes the diagnosis of IPF. ${ }^{21}$ UIP pattern is defined by reticular abnormalities with a subpleural and basal predominance, associated with honeycombing with or without traction bronchiectasis. The most common features of a fibrotic NSIP are relatively 

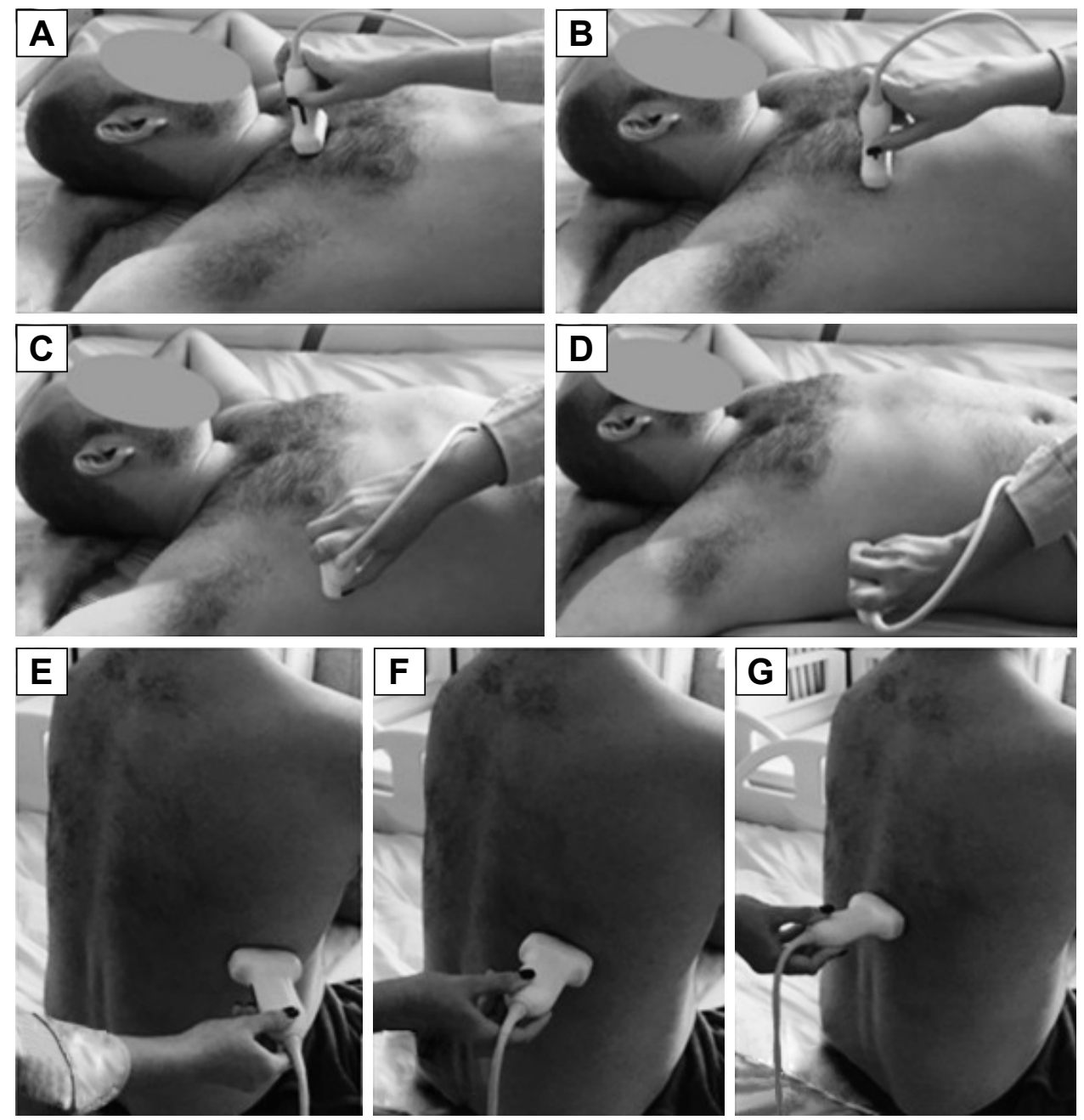

Figure 5 Patient position during LUS, with the probe placed along the (A) paravertebral, (B) midclavicular, (C) anterior axillary, (D) medial axillary, (E) posterior axillary, (F) subscapular, and (G) paravertebral lines.

Abbreviation: LUS, lung ultrasound.

symmetric and bilateral GGOs with associated fine reticulations and pulmonary volume loss resulting in traction bronchiectasis. LUS can differentiate between an alveolar syndrome and an interstitial syndrome, based on the distance and the number of B-lines when it is compared with HRCT features. When the thickness evaluation of the pleura line is associated, LUS can be a good diagnostic imaging tool in IPF assessment.
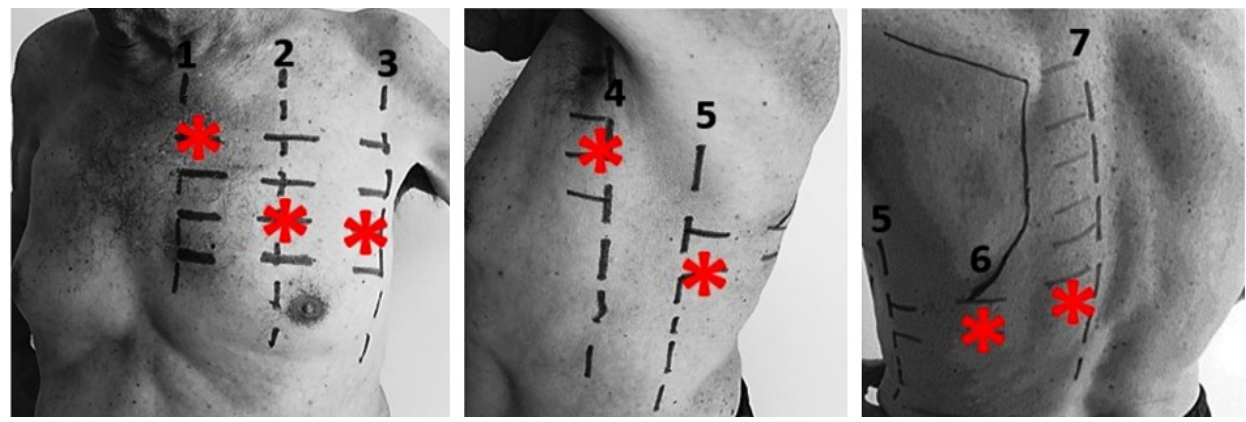

Figure 6 LIS assessment in a reduced LUS protocol (I4 LIS). I: Second IC along parasternal line; 2: fourth IC along midclavicular line; 3: fourth IC along anterior axillary line; 4: fourth IC along medial axillary line; 5: eighth IC along posterior axillary line; 6: eighth IC along subscapular line; 7: eighth IC along paravertebral line. Red asterisks represent places of examination.

Abbreviations: LIS, lung intercostal spaces; LUS, lung ultrasound; IC, intercostal space. 


\section{Case I}

A male, 67 years with IPF, presented with nonproductive cough, dyspnea at rest, and digital clubbing.

HRCT diagnosis (Figure 7) is based on bilateral subpleural reticular opacities in the middle lung, more extensive in the lower lobes with a typical cranial-caudal gradient. Lung architectural distortion is predominant in the lower left lobe, with patchy lobular areas of the normal lung. Associated traction bronchiectasis is seen. Subpleural line of small cystic air with thick walls, defined as honeycombing, is described in the apical and the posterior zones of both lower lobes. ${ }^{51}$

Interstitial syndrome is demonstrated on LUS assessment of both sides of the chest wall. Elementary findings seen beyond the pleura are multiple B-lines generated from the thickened interlobular septa at the lung surface level. They are counted to be $>3$ per IS in both lateral chest regions, and are more numerous in the posterior regions. Also, a thickness of $3.5 \mathrm{~mm}$ with an irregular, fragmented shape of the pleura line is seen in the basal region, correlating with the degree of reticular extension in HRCT imaging.
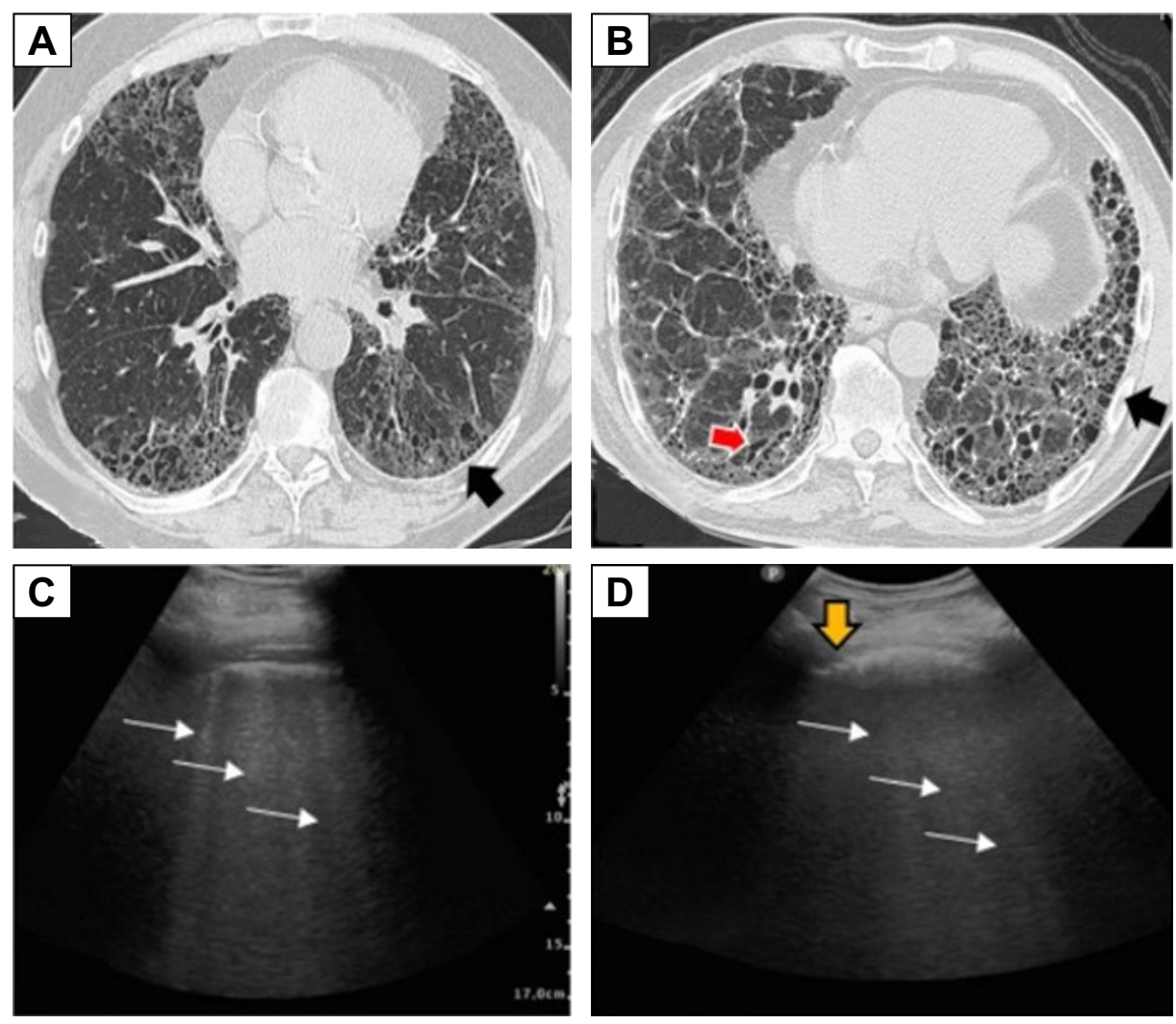

Figure 7 Correlation of HRCT and LUS of a UIP pattern in IPF.

Notes: (A, B) Axial HRCT imaging in the lung window at the level of middle and lower lobes with subpleural reticulation with a basal predominance; honeycombing (black arrows) and traction bronchiectasis (red arrow) associated in $>50 \%$. (C, D) LUS at the level of both sixth PV lines: $>3$ B-lines are seen per IS (white thin arrows) with a median distance between one another of $5.6 \mathrm{~mm}$; irregular, thickened, blurred pleural line confirmed the fibrotic interstitial syndrome. The yellow arrow shows the pleural line. Abbreviations: HRCT, high-resolution computed tomography; LUS, lung ultrasound; NSIP, nonspecific interstitial pattern; IPF, idiopathic pulmonary fibrosis; UIP, usual interstitial pneumonia; PV, paravertebral; IS, intercostal space. 

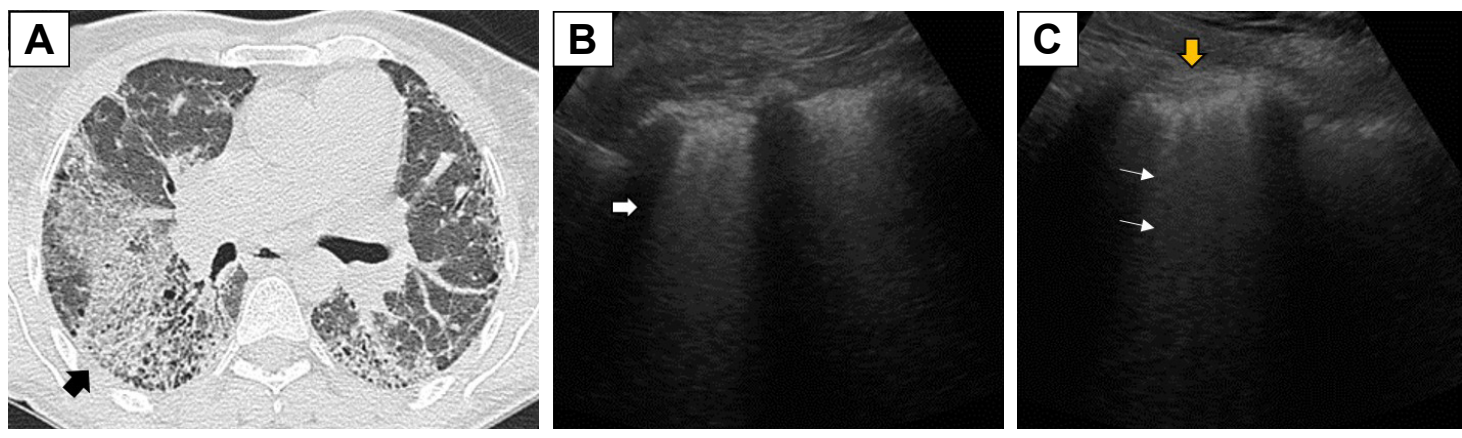

Figure 8 Correlation of HRCT and LUS of an NSIP pattern in a patient with rheumatoid lung disease.

Notes: (A) Axial HRCT imaging in the lung window at the level of middle and lower lobes with extensive GGO (black arrow) and fine reticulation; traction bronchiectasis as a mark of lung volume loss. (B, C) LUS at the level of both eighth SS and PV line: "white lung" (thick white arrow) due to very close multiple B-lines (thin white arrows); irregular, thickened, blurred pleural line (yellow arrow) confirmed the fibrotic interstitial syndrome.

Abbreviations: HRCT, high-resolution computed tomography; LUS, lung ultrasound; NSIP, nonspecific interstitial pattern; PV, paravertebral; SS, subscapular; GGO, ground glass opacity.

associated with a thick, irregular pleura line, the interstitial syndrome is the common substrate US feature in all ILDs. Although it has a specificity of $50 \%$, LUS is a very sensitive, noninvasive, and non-irradiant screening tool to differentiate between upper and basal predominance of the fibrotic changes, and therefore between an IPF and a secondary cause of ILD when it is correlated with HRCT and clinical findings.

\section{Case 3}

A female, 59 years, with chronic hypersensitivity pneumonitis, with a history of mold exposure, presented with dyspnea at rest and dry cough.

HRCT reveals areas of subpleural reticulation (Figure 9) with an upper and middle lung predominance, associated with traction bronchiectasis and bronchiolectasis. Small subpleural cysts representing mild honeycombing are seen in the upper lobes. Lobular areas of decreased attenuation with a mosaic pattern are visualized as indirect signs of bronchial obstruction. The pattern is inconsistent with UIP.

Multiple B-lines as a sonographic marker of interstitial syndrome are the evident findings in the anterior PS and midclavicular zones and also in the AA and middle axillary chest zones. A marked irregular, thickened pleura line due to subpleural fibrotic changes is also present. A lack of B-lines is observed in the inferior parts of posterior regions, with a thin pleura line and horizontal A-lines as signs of normal subpleural space (Figure 10).

\section{LUS could be a reliable tool to differentiate between UIP and a possible UIP pattern}

Subpleural reticulation with a basal predominance is the HRCT common feature for both UIP and possible UIP pattern.
The presence of honeycombing with or without traction bronchiectasis is mandatory for the UIP pattern. ${ }^{18}$

\section{Case 4}

A male patient, 52 years, presented with nonproductive cough, dyspnea on exertion, without any history of exposure or clinical features of known causes of second ILD, with a biopsy-proven IPF.

Mild HRCT reticulation in a subpleural distribution is seen in the lower lobes with traction bronchiectasis. Patchy areas of reticulation are visible in the subpleural region of the middle right lobe. These features correlate with the LUS assessment (Figure 11).

Evidence of B-lines and an irregular pleura line are present in both lateral chest regions. Increased number of B-lines is observed at the lung bases with a more thickened, fragmented pleura line, corresponding to the degree of reticulation in HRCT.

A preserved apical lung with a linear, smooth pleural line and A-lines of normal aerated lung was also noticed.

\section{Case 5}

A male patient, 69 years, presented with dyspnea at rest, with a UIP pattern on HRCT and a diagnosis of IPF by exclusion of known causes of ILD. Severe forms of pulmonary fibrosis with reticulation in a subpleural distribution with a cranialcaudal gradient are the HRCT findings (Figure 12) of the disease. Traction bronchiectasis and a layer of subpleural cysts defined as honeycombing without any atypical finding confirm the defined UIP pattern. In all lung zones a thick, irregular, fragmented pleura line is seen, with a more blurry aspect in the posterior zones. On assessing the complete chest wall bilaterally, $>3$ B-lines are observed for each IS, due to 

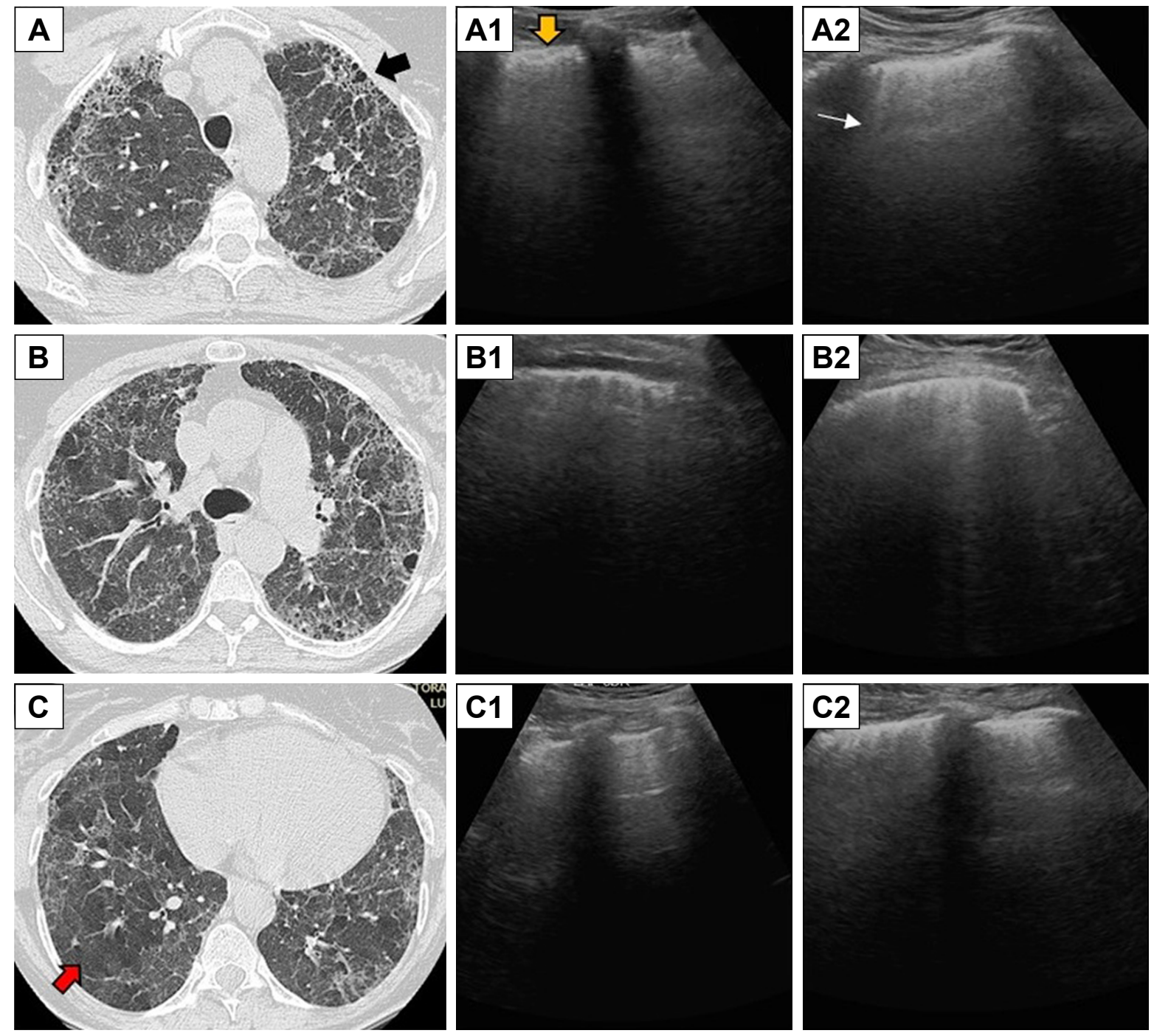

Figure 9 Correlation of HRCT and LUS of an inconsistent UIP pattern in a patient with chronic hypersensitivity pneumonitis.

Notes: (A-C) Axial HRCT imaging in the lung window at the level of upper, middle, and lower lobes with subpleural reticulation; traction bronchiectasis and mild honeycombing (black arrow). Patchy areas of mosaic attenuation due to small bronchial obstruction (red arrow). (AI, A2) LUS: multiple B-lines (thin arrow), with a thick $3.7 \mathrm{~mm}$ pleura line (yellow arrow), are observed in the evaluation of the second IS along PV line on (AI) the right and (A2) the left side; (BI, B2) LUS: B-lines at the fourth IS along MA line on both sides; (CI) LUS: preserved right lung base with thin pleura line and normal A-lines at eighth IS along PV line; (C2) LUS: thin pleura line with a few B-lines and normal A-lines corresponding to mild abnormalities at the eighth IS along SS line.

Abbreviations: HRCT, high-resolution computed tomography; LUS, lung ultrasound; UIP, usual interstitial pneumonia; IS, intercostal space; MA, medial axillary; PV, paravertebral; SS, subscapular.
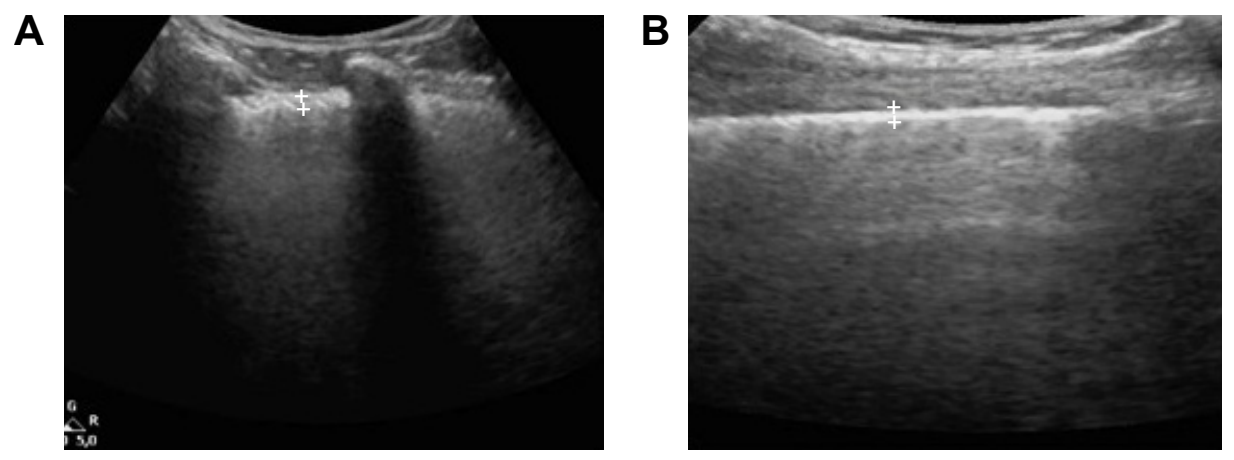

Figure 10 LUS assessment of the thickness of pleura line at the upper (A) versus basal (B) chest regions. Abbreviation: LUS, lung ultrasound. 



Figure I I Correlation of HRCT and LUS in a patient with possible UIP pattern and biopsy-proven idiopathic pulmonary fibrosis.

Notes: (A-C) Axial HRCT imaging in the lung window at the level of upper, middle, and lower lobes with subpleural reticulation and fine traction bronchiectasis with a basal lung predominance (black arrow). No feature of honeycombing. (AI, A2) LUS at the second IS along MC line: normal aerated lung with A-lines (thin white arrow) and smooth pleura line (curve yellow arrow); (BI, B2) LUS at the level of fourth along MA line: a few B-lines (white arrow head) in the lateral lung regions with an irregular pleura line (yellow arrow); (CI, C2) LUS at the level of eighth along PV line: increased B-line (arrowheads) number with thick, irregular pleura line.

Abbreviations: HRCT, high-resolution computed tomography; LUS, lung ultrasound; UIP, usual interstitial pneumonia; IS, intercostal space; MA, medial axillary; PV, paravertebral; MC, midclavicular.

thickened subpleural interlobular septa. A thickened, blurred pleura line and increased number of B-lines are associated with a more extensive fibrotic change in the lower lobes.

The total number of B-lines, and the thickness and the shape of the pleura line are correlated with the extension of pulmonary fibrosis on HRCT (Figure 13), being an LUS marker of severity ${ }^{30}$ The blurry shape of the pleura line is associated with a severe form of subpleural fibrosis associated with subpleural cysts.

\section{Limitation of the method}

LUS is an affordable, non-irradiating method and can be used as a powerful diagnostic tool; however, cautious use is recommended due to its limitations.
These limitations may include the examiner's experience (we recommend a specific training for the operator). There may be variations depending on the used equipment (see "Correlations between LUS and HRCT in the evaluation of pulmonary fibrosis"). It is also important to adapt the probe and frequency to the area of interest (eg, using a highfrequency linear probe for visualization and characterization of the pleural line). It is important for the results to be integrated into the clinical context.

\section{Conclusion}

LUS is an important tool to screen IPF patients because it is a noninvasive, totally radiation-free procedure, and has the ability to differentiate ILDs regarding the distribution and the 

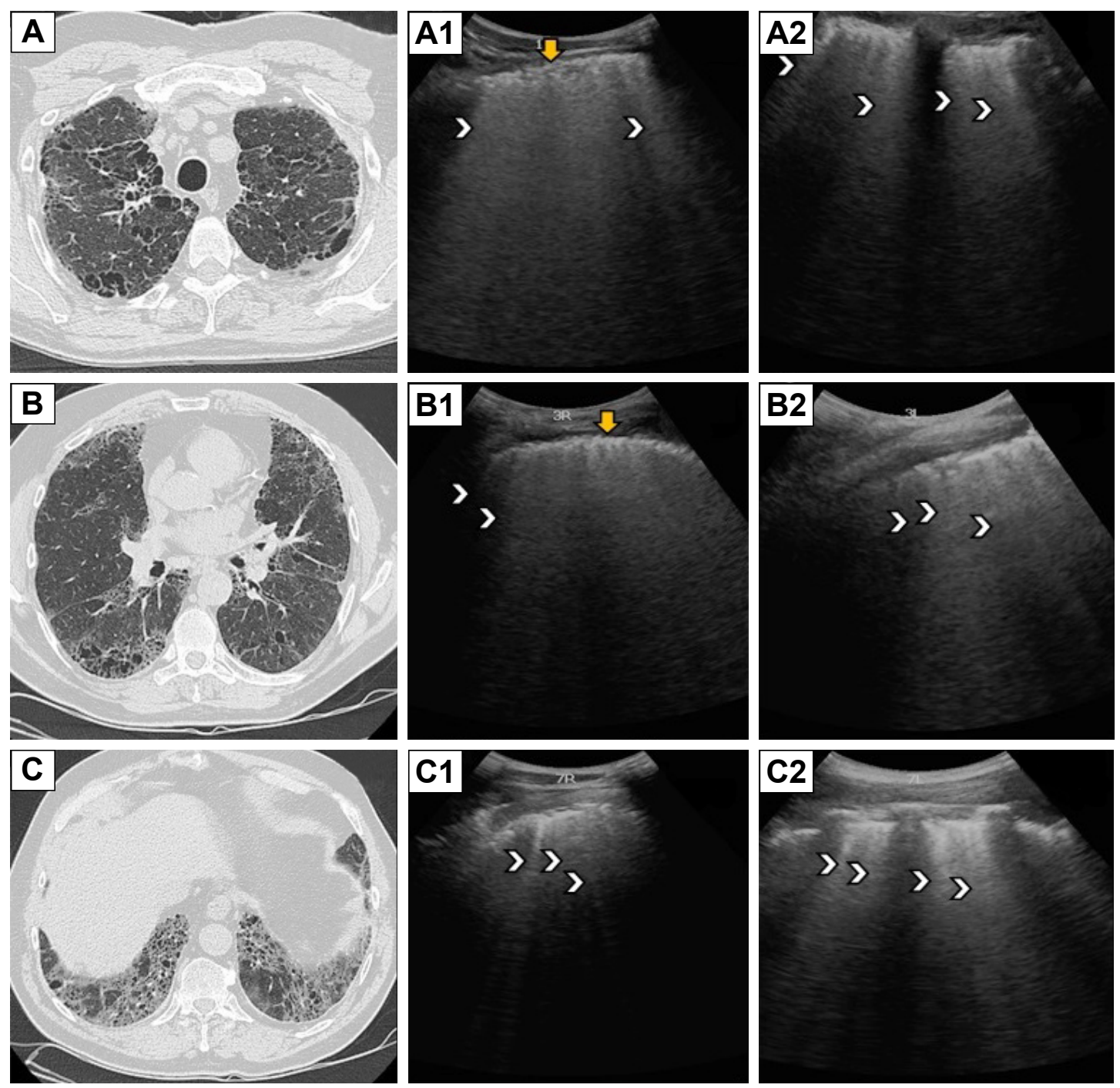

Figure 12 Correlation of HRCT and LUS in a patient with IPF.

Notes: (A-C) Axial HRCT imaging in the lung window at the level of upper, middle, and lower lobes with moderate to severe UIP pattern defined by extensive subpleural areas of reticulation with honeycombing and traction bronchiectasis. LUS images: bilateral multiple B-lines (arrow head) with thick, irregular, blurred pleura line (yellow arrow) in all zones: (AI, A2) at the level of second IS along MC line; (BI, B2) at the level of sixth IS along MA line; (CI, C2) at the level of eighth PV line.

Abbreviations: HRCT, high-resolution computed tomography; LUS, lung ultrasound; UIP, usual interstitial pneumonia; IPF, idiopathic pulmonary fibrosis; IS, intercostal space; MA, medial axillary; PV, paravertebral; MC, midclavicular.
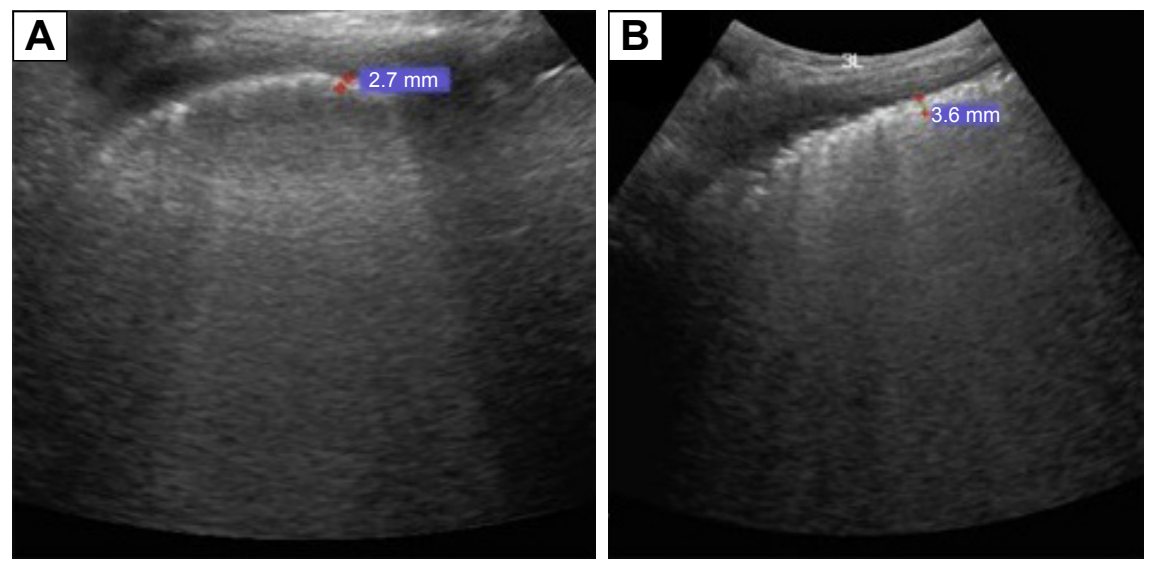

Figure 13 The thickness of pleura line in a patient with mild (A) versus severe (B) IPF. Abbreviation: IPF, idiopathic pulmonary fibrosis. 
number of B-lines and therefore exclude some inconsistent UIP. This is a useful imaging technique in the assessment of early signs of fibrotic interstitial syndrome. If the degree of lung fibrotic LUS index is correlated with HRCT fibrotic index, then LUS might be useful in monitoring the treatment outcome in IPF patients. Further studies are warranted on this topic.

\section{Acknowledgment}

All patients provided written informed consent for their images to be published.

\section{Disclosure}

The authors report no conflicts of interest in this work.

\section{References}

1. Raghu G, Collard HR, Egan JJ, et al; ATS/ERS/JRS/ALAT Committee on Idiopathic Pulmonary Fibrosis. An official ATS/ERS/JRS/ ALAT statement: idiopathic pulmonary fibrosis: evidence-based guidelines for diagnosis and management. Am J Respir Crit Care Med. 2011;183(6):788-824.

2. King TE Jr, Pardo A, Selman M. Idiopathic pulmonary fibrosis. Lancet. 2011;378(9807):1949-1961.

3. Meltzer EB, Noble PW. Idiopathic pulmonary fibrosis. Orphanet $J$ Rare Dis. 2008;3:8.

4. Collard HR, King TE, Bartelson BB, Vourlekis JS, Schwarz MI, Brown KK. Changes in clinical and physiologic variables predict survival in idiopathic pulmonary fibrosis. Am J Respir Crit Care Med. 2003;168(5):538-542.

5. Latsi PI, du Bois RM, Nicholson AG, et al. Fibrotic idiopathic interstitial pneumonia: the prognostic value of longitudinal functional trends. Am J Respir Crit Care Med. 2003;168(5):531-537.

6. King TE Jr, Tooze JA, Schwarz MI, Brown KR, Cherniack RM. Predicting survival in idiopathic pulmonary fibrosis: scoring system and survival model. Am J Respir Crit Care Med. 2001;164(7):1171-1181.

7. Baumgartner KB, Samet JM, Stidley CA, Colby TV, Waldron JA. Cigarette smoking: a risk factor for idiopathic pulmonary fibrosis. $\mathrm{Am} \mathrm{J}$ Respir Crit Care Med. 1997;155(1):242-248.

8. Selman M, Buendía-Roldán I, Pardo A. Aging and pulmonary fibrosis. Rev Invest Clin. 2016;68(2):75-83.

9. American Thoracic Society, European Respiratory Society. American Thoracic Society/European Respiratory Society international multidisciplinary consensus classification of the idiopathic interstitial pneumonias. Am J Respir Crit Care Med. 2002;165(2):277-304.

10. Travis WD, Costabel U, Hansell DM, et al. An official American Thoracic Society/European Respiratory Society statement: update of the international multidisciplinary classification of the idiopathic interstitial pneumonias. Am J Respir Crit Care Med. 2013;188(6):733-748.

11. Raghu G, Weycker D, Edelsberg J, Bradford WZ, Oster G. Incidence and prevalence of idiopathic pulmonary fibrosis. Am J Respir Crit Care Med. 2006;174(7):810-816.

12. Nadrous HF, Myers JL, Decker PA, Ryu JH. Idiopathic pulmonary fibrosis in patients younger than 50 years. Mayo Clin Proc. 2005;80(1): $37-40$.

13. Coultas DB, Zumwalt RE, Black WC, Sobonya RE. The epidemiology of interstitial lung diseases. Am J Respir Crit Care Med. 1994;150(4): 967-972.

14. Collard HR, King TE Jr, Bartelson BB, Vourlekis JS, Schwarz MI, Brown KK. Changes in clinical and physiologic variables predict survival in idiopathic pulmonary fibrosis. Am J Respir Crit Care Med. 2003;168(5):538-542.
15. Fernandez Perez ER, Daniels CE, Schroeder DR, et al. Incidence, prevalence, and clinical course of idiopathic pulmonary fibrosis: a population-based study. Chest. 2010;137(1):129-137.

16. Martinez FJ, Safrin S, Weycker D, et al. The clinical course of patients with idiopathic pulmonary fibrosis. Ann Intern Med. 2005;142(12 Pt 1): 963-967.

17. King TE Jr, Albera C, Bradford WZ, et al. All-cause mortality rate in patients with idiopathic pulmonary fibrosis. Implications for the design and execution of clinical trials. Am J Respir Crit Care Med. 2014;189(7): 825-831.

18. Devaraj A. Imaging: how to recognise idiopathic pulmonary fibrosis. Eur Respir Rev. 2014;23(132):215-219.

19. European Commission. Radiation Protection 118: Referral Guidelines for Imaging. Luxembourg: Office for Official Publications of the European Communities; 2001. Available from: https://health.gov.mt/ en/forms/Documents/radiation_protection.pdf. Accessed August 1, 2017.

20. Lynch DA, Godwin JD, Safrin S, et al. High-resolution computed tomography in idiopathic pulmonary fibrosis: diagnosis and prognosis. Am J Respir Crit Care Med. 2005;172(4):488-493.

21. Lynch DA, Travis WD, Muller NL, et al. Idiopathic interstitial pneumonias: CT features. Radiology. 2005;236(1):10-21.

22. Sverzellati N. Highlights of HRCT imaging in IPF. Respir Res. 2013; 14(Suppl 1):S3.

23. Walsh SL, Calandriello L, Sverzellati N, Wells AU, Hansell DM. Interobserver agreement for the ATS/ERS/JRS/ALAT criteria for a UIP pattern on CT. Thorax. 2016;71(1):45-51.

24. Lichtenstein D, Hulot JS, Rabiller A, Tostivint I, Meziere G. Feasibility and safety of ultrasound-aided thoracentesis in mechanically ventilated patients. Intensive Care Med. 1999;25(9):955-958.

25. Mathis G. Thoraxsonography - part I: chest wall and pleura. Ultrasound Med Biol. 1997;23(8):1131-1139.

26. Sperandeo M, Filabozzi P, Varriale A, et al. Role of thoracic ultrasound in the assessment of pleural and pulmonary diseases. J Ultrasound. 2008;11(2):39-46.

27. Lichtenstein D. Classification of artefacts. In: Lichtenstein D, editor. Whole Body Ultrasonography in the Critically Ill. Heidelberg, New York, Berlin: Springer-Verlag; 2010:185-188.

28. Volpicelli G. Lung sonography. J Ultrasound Med. 2013;32(1): 165-171.

29. Jambrik Z, Monti S, Coppola V, et al. Usefulness of ultrasound lung comets as a nonradiologic sign of extravascular lung water. Am J Cardiol. 2004;93(10):1265-1270.

30. Buda N, Piskunowicz M, Porzezińska M, Kosiak W, Zdrojewski Z. Lung ultrasonography in the evaluation of interstitial lung disease in systemic connective tissue diseases: criteria and severity of pulmonary fibrosis analysis of 52 patients. Ultraschall Med. 2016;37(4):379-385.

31. Wohlgenannt S, Gehmacher O, Gehmacher U, Kropf A, Mathis G. Sonographic finding in interstitial lung diseases. Ultraschall Med. 2001;22:27-33.

32. Picano E, Frassi F, Agricola E, Gligorova S, Gargani L, Mottola G. Ultrasound lung comets: a clinically useful sign of extravascular lung water. J Am Soc Echocardiogr. 2006;19(3):356-363.

33. Soldati G, Copetti R, Sher S. Sonographic interstitial syndrome: the sound of lung water. J Ultrasound Med. 2009;28(2):163-174.

34. Agricola E, Bove T, Oppizzi M, et al. "Ultrasound comet-tail images": a marker of pulmonary edema: a comparative study with wedge pressure and extravascular lung water. Chest. 2005;127(5):1690-1695.

35. Sperandeo M, Varriale A, Sperandeo G, et al. Transthoracic ultrasound in the evaluation of pulmonary fibrosis: our experience. Ultrasound Med Biol. 2009;35(5):723-739.

36. Lichtenstein D, Meziere G, Biderman P, Gepner A, Barre O. The comettail artifact. An ultrasound sign of alveolar-interstitial syndrome. Am J Respir Crit Care Med. 1997;156(5):1640-1646.

37. Copetti R, Soldati G, Copetti P. Chest sonography: a useful tool to differentiate acute cardiogenic pulmonary edema from acute respiratory distress syndrome. Cardiovasc Ultrasound. 2008;6:16. 
38. Volpicelli G, Elbarbary M, Blaivas M, et al; International Liaison Committee on Lung Ultrasound (ILC-LUS) for International Consensus Conference on Lung Ultrasound (ICC-LUS). International evidencebased recommendations for point-of-care lung ultrasound. Intensive Care Med. 2012;38(4):577-591.

39. Barskova T, Gargani L, Guiducci S, et al. Lung ultrasound for the screening of interstitial lung disease in very early systemic sclerosis. Ann Rheum Dis. 2013;72(3):390-395.

40. Moazedi-Fuerst FC, Kielhauser S, Brickmann K, et al. Sonographic assessment of interstitial lung disease in patients with rheumatoid arthritis, systemic sclerosis and systemic lupus erythematosus. Clin Exp Rheumatol. 2015;33(4 Suppl 91):S87-S91.

41. Hasan AA, Makhlouf HA. B-lines: transthoracic chest ultrasound signs useful in the assessment of interstitial lung diseases. Ann Thorac Med. 2014;9(2):99-103.

42. Gargani L, Doveri M, D'Errico L, et al. Ultrasound lung comets in systemic sclerosis: a chest sonography hallmark of pulmonary interstitial fibrosis. Rheumatology. 2009;48(11):1382-1387.

43. Warrick JH, Bhalla M, Schabel SI, Silver RM. High resolution computed tomography in early scleroderma lung disease. J Rheumatol. 1991; 18(10):1520-1528.

44. Sperandeo M, De Cata A, Molinaro F, et al. Ultrasound signs of pulmonary fibrosis in systemic sclerosis as timely indicators for chest computed tomography. Scand J Rheumatol. 2015;44(5):389-398.
45. Gutierrez M, Salaffi F, Carotti M, et al. Utility of a simplified ultrasound assessment to assess interstitial pulmonary fibrosis in connective tissue disorders - preliminary results. Arthritis Res Ther. 2011;13(4):R134.

46. Cogliati C, Antivalle M, Torzillo D, et al. Standard and pocket-size lung ultrasound devices can detect interstitial lung disease in rheumatoid arthritis patients. Rheumatology. 2014;53(8):1497-1503.

47. Tardella M, Gutierrez M, Salaffi F, et al. Ultrasound in the assessment of pulmonary fibrosis in connective tissue disorders: correlation with high-resolution computed tomography. J Rheumatol. 2012;39(8): 1641-1647.

48. Delle Sedie A, Doveri M, Frassi F, et al. Ultrasound lung comets in systemic sclerosis: a useful tool to detect lung interstitial fibrosis. Clin Exp Rheumatol. 2010;28(5 Suppl 62):S54.

49. Dietrich CF, Mathis G, Blaivas M, et al. Lung B-line artefacts and their use. J Thorac Dis. 2016;8(6):1356-1365.

50. Lichtenstein DA, Mezière GA. Relevance of lung ultrasound in the diagnosis of acute respiratory failure: the BLUE protocol. Chest. 2008; 134(1):117-125

51. Hansell D, Bankier AA, MacMahon H, McLoud TC, Müller NL, Remy J. Fleischner Society: glossary of terms for thoracic imaging. Radiology. 2008;246(3):697-722.
Clinical Interventions in Aging

\section{Publish your work in this journal}

Clinical Interventions in Aging is an international, peer-reviewed journal focusing on evidence-based reports on the value or lack thereof of treatments intended to prevent or delay the onset of maladaptive correlates of aging in human beings. This journal is indexed on PubMed Central, MedLine,

\section{Dovepress}

CAS, Scopus and the Elsevier Bibliographic databases. The manuscript management system is completely online and includes a very quick and fair peer-review system, which is all easy to use. Visit http://www.dovepress. com/testimonials.php to read real quotes from published authors. 